A. M. Wessels $\cdot$ S. Simsek $\cdot$ P. L. Remijnse

D. J. Veltman - G. J. Biessels • F. Barkhof •

P. Scheltens • F. J. Snoek • R. J. Heine •

S. A. R. B. Rombouts

\title{
Voxel-based morphometry demonstrates reduced grey matter density on brain MRI in patients with diabetic retinopathy
}

Received: 9 November 2005 / Accepted: 29 March 2006 / Published online: 16 May 2006

C) Springer-Verlag 2006

\begin{abstract}
Aims/hypothesis: In addition to nephropathy, retinopathy and peripheral neuropathy, a microvascular complication of type 1 diabetes that may be tentatively referred to as 'diabetic encephalopathy' has gained increasing attention. There is growing evidence that lowered cognitive performance in patients with type 1
\end{abstract}

A. M. Wessels $(\bowtie) \cdot$ F. J. Snoek

Department of Medical Psychology,

VU University Medical Center,

Van der Boechorststraat 7,

1081 BT Amsterdam, The Netherlands

e-mail: am.wessels@vumc.nl

Tel.: +31-020-4448224

Fax: +31-020-4448230

S. Simsek · R. J. Heine

Department of Endocrinology/Diabetes Center,

VU University Medical Center,

Amsterdam, The Netherlands

P. L. Remijnse · D. J. Veltman

Department of Psychiatry, VU University Medical Center,

Amsterdam, The Netherlands

F. Barkhof

Department of Radiology, VU University Medical Center, Amsterdam, The Netherlands

P. Scheltens

Department of Neurology, VU University Medical Center, Amsterdam, The Netherlands

S. A. R. B. Rombouts

Department of Physics and Medical Technology,

VU University Medical Center,

Amsterdam, The Netherlands

G. J. Biessels

Department of Neurology, University Medical Center Utrecht,

Utrecht, The Netherlands

S. A. R. B. Rombouts

Department of Radiology, Leiden University Medical Center,

Leiden, The Netherlands

S. A. R. B. Rombouts

Department of Psychology, Leiden University,

Leiden, The Netherlands diabetes is related to chronic hyperglycaemia rather than recurrent episodes of severe hypoglycaemia, as previously speculated. The aim of our study was to use magnetic resonance imaging (MRI) to establish whether long-term hyperglycaemia, resulting in advanced retinopathy, contributes to structural changes in the brain (reduced grey matter). Subjects, materials and methods: We applied voxel-based morphometry on magnetic resonance images to compare grey matter density (GMD) between three groups of participants. GMD is used as a marker of cortical atrophy. We compared 13 type 1 diabetic patients with a microvascular complication (i.e. proliferative retinopathy) with 18 type 1 diabetic patients who did not have retinopathy in order to assess the effects of microvascular changes on GMD. Both patient groups were compared with 21 healthy control subjects to assess the effect of diabetes in itself. Results: Patients with diabetic retinopathy showed reduced GMD in the right inferior frontal gyrus and right occipital lobe compared both with patients without retinopathy and with healthy controls $(p<0.05)$. Conclusions/interpretation: Our data show that patients with type 1 diabetes, who, as a consequence of chronic hyperglycaemia, had developed advanced retinopathy, also showed increased focal cortical atrophy on brain MRI.

Keywords Brain MRI - Grey matter density · Microvascular complications - Type 1 diabetes

Abbreviations DRP: diabetic retinopathy - GMD: grey matter density - MRI: magnetic resonance imaging · NDRP: non-diabetic retinopathy - SPM: statistical parametric mapping $\cdot$ VBM: voxel-based morphometry

\section{Introduction}

In addition to the well-known and well-described microvascular complications of diabetes, i.e. nephropathy, retinopathy and peripheral neuropathy, a condition that may be tentatively referred to as 'diabetic encephalopathy' is attracting more attention. This term was introduced in 
1965 [1] to describe the pathological changes in the central nervous system of young patients with long-term type 1 diabetes mellitus. The relationship between the development of this encephalopathy and exposure to chronic hyperglycaemia, leading to microangiopathy, is not well established. A recent meta-analysis concluded that lowered cognitive performance of patients with type 1 diabetes appears not to be associated with recurrent episodes of severe hypoglycaemia, as often speculated, but rather with microvascular complications such as retinopathy [2]. A longitudinal study [3] showed that cognitive efficiency may decline over time in diabetic adults, and that this neurocognitive change may be linked, at least in part, to the occurrence of microvascular complications such as proliferative retinopathy and to elevated blood pressure. Other evidence for a damaging effect of chronic hyperglycaemia on the brain comes from the work of Ferguson et al. [4]. Subjects who had background retinopathy performed less well across most cognitive domains examined. The authors also found that background retinopathy was associated with small punctate white matter lesions corresponding to enlarged perivascular spaces.

To date, the few neuroradiological studies that have assessed structural brain abnormalities in patients with type 1 diabetes have demonstrated conflicting results [4-8]. The majority of these studies have been based on manual or semi-automated region-of-interest-guided measurements, which may be inherently biased.

This prompted us to employ a fully automated, sensitive, standardised, whole-brain technique known as voxel-based morphometry (VBM) [9], applying it to magnetic resonance images to study structural differences in cortical grey matter density (GMD) in patients with type 1 diabetes. Our aim was to establish whether long-term hyperglycaemia resulting in advanced retinopathy was associated with reduced GMD in the brain. We used advanced retinopathy as a marker of microvascular disease because the retinal vessels share common anatomical, embryological and physiological characteristics with the cerebral microcirculation $[10,11]$. Abnormalities of the retinal arterioles (e.g. microaneurysms, retinal haemorrhages and arteriolar narrowing) may be markers of concomitant cerebral small vessel diseases [12].

We therefore included patients with type 1 diabetes and proliferative retinopathy, and compared these patients with type 1 diabetic patients who did not have retinopathy in order to assess the relation between microvascular changes and GMD. Both patient groups were compared with healthy controls to assess the effect of diabetes in itself.

We hypothesised that patients with retinopathy have reduced GMD compared with patients without retinopathy and healthy controls.

\section{Subjects, materials and methods}

\section{Subjects}

Three age- and sex-matched groups of participants were included: 18 patients with type 1 diabetes (World Health
Organization criteria, 1999) without microvascular complications (non-diabetic retinopathy [NDRP], maximum of three microaneurysms), 13 patients with type 1 diabetes with a microvascular complication, i.e. diabetic proliferative retinopathy (DRP) (grade 5 DRP according to the EURODIAB classification [13]) and 21 healthy controls (Table 1). All subjects were right-handed and normotensive $(<140 / 90 \mathrm{mmHg})$. None of the NDRP patients had a microor macrovascular diabetic complication. Four DRP patients were known to have nephropathy and two other patients were known to have nephropathy and neuropathy. Exclusion criteria were: previous alcohol or drug abuse; a history of psychiatric disease/treatment; a history of severe head trauma accompanied by loss of consciousness; stroke; epilepsy; and a history of severe, recurrent hypoglycaemia (defined as more than five episodes that required external assistance for recovery) [14]. Written consent was obtained from all subjects and the study was approved by the local medical ethics advisory committee. The investigations were carried out in accordance with the principles of the Declaration of Helsinki as revised in 2000.

\section{Magnetic resonance image acquisition}

Imaging was performed on a $1.5 \mathrm{~T}$ Siemens Sonata (Siemens, Erlangen, Germany) scanner using a standard circularly polarised head coil, with foam padding to restrict head motion. A localiser scan was first performed for positioning of the image planes, followed by an automated shim procedure to improve magnetic field homogeneity. Scans were obtained as whole-brain T1-weighted MPRAGE (magnetisation prepared rapid acquisition gradient echo) volumes and were acquired in the coronal plane (inversion time $950 \mathrm{~ms}$, repetition time 2,700 ms; echo time $5.15 \mathrm{~ms}$; flip angle $8^{\circ}$; 160 slices; voxel size $1 \times 1 \times 1.5 \mathrm{~mm}$ ).

This imaging protocol was part of a functional magnetic resonance imaging (MRI) study which did not include a fluid-attenuating inversion recovery sequence. For this reason, white matter lesions could not be scored.

Table 1 Characteristics of participants

\begin{tabular}{lllll}
\hline & $\begin{array}{l}\text { No retinopathy } \\
(\text { NDRP) }\end{array}$ & $\begin{array}{l}\text { Retinopathy } \\
(\text { DRP) }\end{array}$ & $\begin{array}{l}\text { Healthy } \\
\text { subjects }\end{array}$ & $p$ \\
\hline$n$ & 18 & 13 & 21 & \\
Age (years) & $39.8 \pm 6.3$ & $41.7 \pm 5.5$ & $36.3 \pm 7.9$ & 0.07 \\
Sex (males:females) & $7: 11$ & $5: 8$ & $7: 14$ & 0.93 \\
HbA $_{1 \mathrm{c}}$ (\%) & $7.9 \pm 1.12$ & $8.1 \pm 1.13$ & NA & 0.74 \\
$\begin{array}{l}\text { Duration of diabetes } \\
\text { (years) }\end{array}$ & $23.7 \pm 9.4$ & $29.9 \pm 7.1$ & NA & 0.06 \\
$\begin{array}{l}\text { Age at diagnosis } \\
\text { (years) }\end{array}$ & $16.6 \pm 7.9$ & $10.9 \pm 4.7$ & NA & 0.06 \\
\hline
\end{tabular}

Data are mean $\pm \mathrm{SD}$

${ }^{\text {a }}$ Two pictures were made of the retina of each eye, one with the macula and one with the optic disc in the centre (using a Topcon non-mydriatic camera)

$N A$ Not applicable 
Statistical analysis

Statistical analysis was performed using SPSS version 11.0 (SPSS, Chicago, IL, USA). Demographic data of patients and healthy controls were analysed using one-way ANOVA for continuous variables and the $\chi^{2}$ test for binomial variables.

\section{MRI data analysis}

Data analysis was performed using statistical parametric mapping software (SPM2; Wellcome Department of Cognitive Neurology, Institute of Neurology, London, UK, http:/www.fil.ion.ucl.ac.uk/spm/) with the VBM tool Jena script http://dbm.neuro.uni-jena.de/vbm.html; both websites accessed on 9 January 2006) in MATLAB version 6 (Mathworks, Natick, MA, USA).

We used optimised VBM with a custom template and priors because this method improves the plausibility of intergroup comparisons, presumably because of improved segmentation and spatial normalisation $[15,16]$.

Briefly, the processing steps outlined by this protocol are: (1) the creation of a customised anatomical T1weighted template and prior probability images separately for each group, by normalising the brain images to the default SPM T1-weighted template, segmenting, averaging and smoothing the averaged brains in each group; and (2) the normalisation of the structural brain images in each group using these customised templates, segmenting and cleaning the original T1-weighted images. This procedure has been described in detail previously [16].

Statistical analysis used the general linear model and is based on the random Gaussian field theory [17]. We performed analyses on the smoothed grey matter images only. Differences in GMD between the three groups were examined using one-way ANOVA and post hoc comparisons. We calculated the positive contrasts (controls $>$ NDRP + DRP; controls > NDRP; controls > DRP and NDRP $>$ DRP) and the negative/inverse contrasts. Results were thresholded at $p<0.05$ corrected for multiple comparisons at the cluster level.

This analysis was based on a strict $p$ value $(p<0.05$, cluster-corrected). In a second analysis, we restricted the analysis to regions of interest which followed from one of the contrasts (see Results), using less stringent statistics $(p<0.001$ uncorrected). Thus, this procedure limited statistical comparisons in SPM to the desired anatomical region and increased sensitivity (lower $p$ value) while controlling for false positives (restricted to certain regions).

Additionally, multiple regression analyses between GMD and $\mathrm{HbA}_{1 \mathrm{c}}$, diabetes duration, onset of diabetes and blood pressure were performed, adjusting for age, to identify clusters of voxels whose GMD related to these parameters. A threshold of $p<0.05$ was used, corrected for multiple comparisons at the cluster level.

\section{Results}

\section{Controls vs NDRP and DRP groups}

There were no significant differences in GMD between healthy volunteers and type 1 diabetes patients (NDRP and DRP groups combined).

\section{NDRP group vs DRP group}

Reduced GMD was observed in the DRP group compared with the NDRP group in the left middle frontal gyrus $(Z=3.86)$, right inferior frontal gyrus $(Z=4.43)$, right occipital lobe $(Z=4.36)$ and left cerebellum $(Z=4.08)$ (Fig. 1). Table 2 lists the stereotaxic coordinates of the most significant clusters of voxels.

Differences between healthy volunteers and the NDRP group and the DRP group in these same regions were not statistically significant, although GMDs tended to be reduced in the DRP group relative to controls.
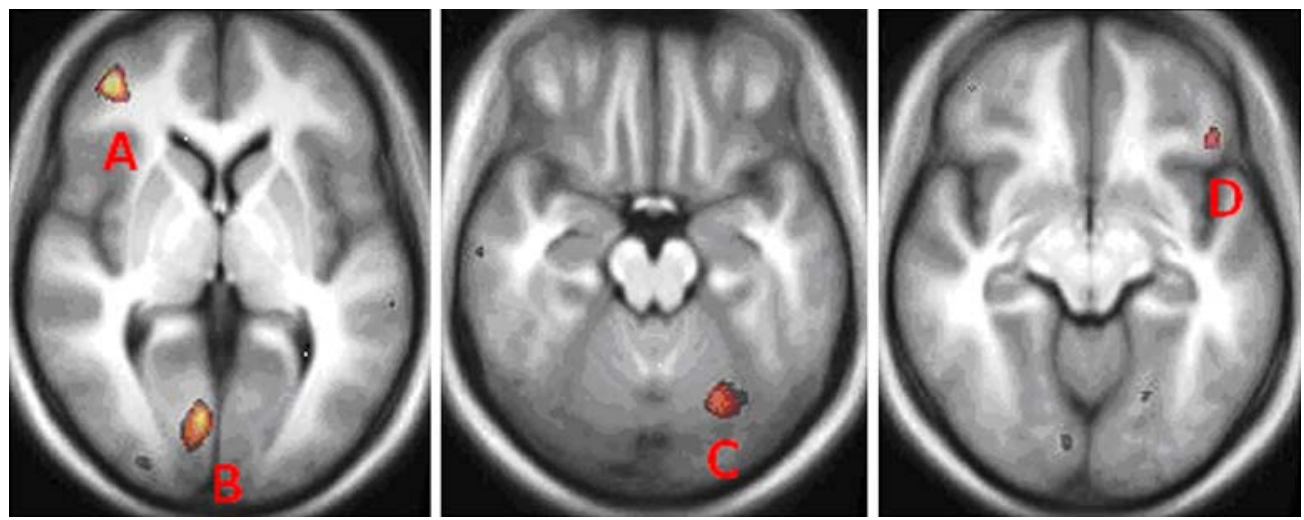

Fig. 1 Voxel-based morphometry comparisons between 13 patients with type 1 diabetes and proliferative retinopathy (DRP) and 18 patients with type 1 diabetes without retinopathy (NDRP). Right and left are inverted in the image, so the right side of the brain appears on the left side in the image. Changes in GMD are shown on a mean normalised structural image in axial planes. Significantly reduced GMD is seen in the right frontal gyrus (marked A), right occipital lobe (marked B), the left cerebellum (marked C) and left middle frontal gyrus (marked D) in the DRP group compared with the NDRP group ( $p<0.05$ corrected for multiple comparisons) 
In the second analysis we calculated the following contrasts: controls $>$ NDRP (mask NDRP $>$ DRP) and controls $>$ DRP (mask NDRP $>$ DRP). The former contrast did not show significant differences in GMD between the groups. The latter, however, displayed significant reductions at the voxel level $(p<0.001)$ of grey matter in the DRP group compared with controls in the right inferior frontal gyrus $(Z=3.19)$ and right occipital lobe $(Z=3.77)$. Examination of the inverse contrasts, i.e. reduced GMD of controls compared with DRP and NDRP and reduced GMD of NDRP compared with DRP, did not yield any significant results for the analyses mentioned above.

We observed no significant association between atrophy and $\mathrm{HbA}_{1 \mathrm{c}}$, diabetes duration, onset of diabetes or blood pressure within the type 1 diabetes group. These variables did not account for the differences in GMD between the DRP and the NDRP groups.

\section{Discussion}

We found that the DRP patients, compared with the NDRP patients, showed reduced GMD in the right inferior frontal gyrus, right occipital lobe, left cerebellum and left middle frontal gyrus. Two of these regions, the right inferior frontal gyrus and the right occipital lobe, also showed reduced GMD in comparison with the healthy participants. The NDRP group did not show reduced GMD compared with healthy participants.

Central and peripheral atrophy in type 1 diabetes $[7,8$, 18] and subcortical white matter lesions [4, 5] have been reported in the literature. The methods used in these studies are semiquantitative. In the study by Ferguson and colleagues [4], a relationship with retinopathy was observed. However, they used several scoring systems to capture the intensity, distribution and appearance of white matter lesions, because no single scale was judged to be an adequate summary. Moreover, small punctate white matter lesions, which represent enlarged perivascular spaces, were scored on a scale of $0-3$, because these lesions were not accounted for by any of the above rating scales.

The strength of our study lies in the inclusion of matched non-diabetic controls and the use of a sensitive, fully automated whole-brain technique that detects regionally specific differences in brain tissue composition on a voxelby-voxel basis. At its simplest, it involves a voxelwise comparison of the local concentration of grey matter between groups of subjects [9].
Recently, an interesting paper on the effects of type 1 diabetes on GMD as measured by VBM was published [19]. This study investigated whether lower grey matter densities in patients with type 1 diabetes were present, and if so, whether they were associated with glycaemic control and/or a history of severe hypoglycaemic events. It was found that, compared with healthy controls, patients with type 1 diabetes showed lower GMD in several brain areas. One or more severe hypoglycaemic events was associated with less GMD in the left cerebellar posterior lobe. Moreover, the higher the $\mathrm{HbA}_{1 \mathrm{c}}$ level (suggesting compromised glycaemic control) the lower the GMD concentration, including the left posterior cingulate, right parahippocampal gyrus, left hippocampus, left superior temporal gyrus, right occipital cuneus and left thalamus. In an additional (post hoc) analysis, the authors found that a greater degree of retinopathy was associated with greater GMD loss in brain regions used for cognition (frontal and temporal regions). It was concluded that persistent hyperglycaemia may be associated with lower levels of GMD in patients with type 1 diabetes and that the superior temporal gyrus is particularly vulnerable to the effects of diabetes. Although this study and our study found a relation between retinopathy and the degree of density reduction, there are important differences in design and patient population that complicate comparison of results. Whereas our study was designed as a case-control study to test the effect of microangiopathy on the brain, the study by Musen et al. [19] investigated whether GMD reduction occurred in type 1 diabetic patients, and, if so, whether hypoglycaemia and/or longstanding hyperglycaemia were associated with reduced GMD. Overall, the effects we found were less significant, probably because our initial analysis was based on a stricter statistical threshold $(p<0.05$ corrected for multiple comparisons). Lowering our threshold would probably reveal more significant reductions in GMD. We did not indicate the magnitude of the GMD reduction, but comparing $Z$-scores indicates that the effects found are in fact of the same magnitude (i.e. both studies found subtle effects).

Our results support the notion that type 1 diabetes is associated with modest regional cortical atrophy, but these changes are limited to patients with advanced microvascular complications of diabetes. From the results of the regression analysis (no significant association between atrophy and $\mathrm{HbA}_{1 \mathrm{c}}$, diabetes duration, onset of diabetes or blood pressure within the type 1 diabetes group and between the DRP and NDRP groups), one might conclude that the GMD differences found in patients with type 1

Table 2 Areas of grey matter density reduction in the DRP group vs the NDRP group

\begin{tabular}{|c|c|c|c|c|c|}
\hline \multicolumn{3}{|c|}{ Coordinates $x, y, z(\mathrm{~mm})$} & \multirow{2}{*}{$\frac{\text { Cluster level }}{0.009}$} & \multirow{2}{*}{$\frac{Z \text {-score }}{4.43}$} & \multirow{2}{*}{$\begin{array}{l}\text { Anatomical location } \\
\text { Right inferior frontal gyrus }\end{array}$} \\
\hline 40 & 49 & 2 & & & \\
\hline 12 & -80 & 7 & 0.001 & 4.36 & Right occipital lobe \\
\hline-42 & 43 & 24 & 0.046 & 3.86 & Left middle frontal gyrus \\
\hline
\end{tabular}

Cut-off $p<0.05$ at cluster level 
Fig. 2 Possible mechanism underlying reduced grey matter density in the DRP patient group

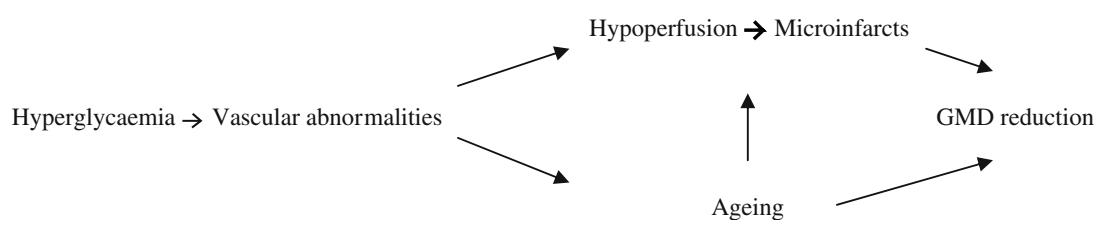

diabetes are associated with long-term hyperglycaemic end-organ damage instead of more short-term indices of hyperglycaemia itself, such as $\mathrm{HbA}_{1 \mathrm{c}}$. The question arises whether some individuals are more sensitive to hyperglycaemic damage (and will therefore develop retinopathy and brain atrophy), or that the GMD reductions we see are indeed the result of prolonged exposure to hyperglycaemia in the past. To answer this question, a study with a longitudinal design is warranted.

Our results indicate that DRP patients, compared with NDRP patients, showed reduced GMD in four brain areas (left middle frontal gyrus, $Z=3.86$; right inferior frontal gyrus, $Z=4.43$; right occipital lobe, $Z=4.36$; left cerebellum, $Z=4.08$ ).

The inclusion of a matched healthy control group is an important strength of our study, allowing us to test whether reduced GMD is associated with diabetes in itself or with the presence of microvascular disease. If reduction of GMD occurs as a result of diabetes (other than microvascular disease), one would expect that the differences in GMD reduction between the DRP and the NDRP group would be less pronounced than those between the DRP group and healthy controls. In a second analysis we investigated whether the two patient groups differed from the healthy controls, applying a less stringent threshold of $p<0.001$ (uncorrected for multiple comparisons; a widely accepted and used statistical threshold) to increase sensitivity to group differences, while restricting our analysis to predefined regions of interest. Only voxels that were found to be 'active' in a previous contrast were queried (in this case, we used the left middle frontal gyrus, the right inferior frontal gyrus, the right occipital lobe and the left cerebellum as regions of interest for further analysis). We found significant reductions at the voxel level $(p<0.001)$ of GMD in the DRP group compared with the healthy participants in the right inferior frontal gyrus $(Z=3.19)$ and the right occipital lobe $(Z=3.77)$. We did not find differences between the healthy participants and the NDRP group in this analysis. The Z-scores in the NDRPDRP comparison (initial analysis) are somewhat higher than the $Z$-scores in the comparison of the DRP group with the healthy participants (the second, less stringent analysis), The $Z$-scores of this latter comparison did not reach significance with the initial, stringent thresholding, but were not statistically lower than the $Z$-scores of the DRP-NDRP comparison (which did reach significance in the initial analysis). Hence, we can conclude that the DRP group showed significantly reduced GMD compared with the NDRP group and compared with the healthy controls, and that the differences between the DRP and the NDRP groups were not greater than the differences between the DRP group and the healthy participants. Furthermore, because we did not find differences between the NDRP group and the healthy participants, we conclude that the reduction in GMD is not related to diabetes in itself, but rather has a microvascular origin.

The mechanism behind the association between retinopathy and reduced GMD is unknown, but hyperglycaemia is likely to be an important mediator in both. Hyperglycaemia may lead to an accumulation of potentially toxic glucose metabolites, oxidative stress, accelerated formation of advanced glycation end-products and microvascular changes in the brain, analogous to those observed in peripheral neuropathy [20]. Considering vascular changes in the brain in diabetes, it is known that structural abnormalities at the microvascular level include thickening of capillary basement membranes and decreased capillary density [21]. During ageing, brain capillaries undergo progressive degeneration, as evidenced by amyloid deposition, thickened basement membranes and reduced vessel elasticity [22, 23]. Considering the similarities of these events, it is possible that diabetes and ageing share the same pathogenic processes. Since only the DRP group showed a reduction in GMD, it is likely that hyperglycaemia-induced changes contribute to accelerated ageing of the brain, as reflected in increased cortical atrophy. However, there is evidence that accelerated ageing is associated with global grey matter volume decreases rather than only with local areas of accelerated loss $[15,24]$. The fact that we did not find a global ageing effect can probably be attributed to below-threshold effects, but this needs to be clarified in future research.

Another possible contributing mechanism underlying the diminished GMD in the DRP group may be (recurrent) microinfarction in the grey matter, resulting in its loss. Microinfarction has been reported as the basis of dementia $[25,26]$; it is associated with a history of transient ischaemic attacks [27] and contributes significantly to the progression of cognitive deficits in brain ageing [28]. The inferior frontal gyrus and the occipital lobe, showing decreased GMD in the DRP group compared with the NDRP group and the healthy controls, are the watershed areas of the medial cerebral artery and the posterior cerebral artery. Watershed areas are located peripherally to primary distribution areas of the vascular systems. Because they are located at the extreme ends of the arterial distribution, they are particularly vulnerable to ischaemia and infarction in people with circulation problems. It is well established that vascular watershed zones are the first to be deprived of sufficient blood flow in the event of cerebral hypoperfusion [29]. Therefore, we suggest that the restriction of cortical microinfarcts in our population to watershed cortical areas points to cerebral hypoperfusion (resulting from hyperglycaemia-induced vascular abnor- 
malities) as the determining factor in their pathogenesis. It is possible that diabetes patients with a microvascular complication have an additional risk of developing a reduction in GMD, since both ageing and hypoperfusion can affect the integrity of the grey matter (Fig. 2).

Further information about the occurrence and severity of watershed cortical infarcts, and hypoperfusion as a possible determinant of these infarcts with respect to the progression and severity of diabetes is needed.

The possible impact of isolated microvascular lesions on cognition remains controversial, and data regarding this issue are very scarce. Although some studies point to a possible causal relationship with dementia [30-33], others challenge this point of view [34]. Shrinkage and atrophy of the brain are generally considered to be a risk factor for cognitive decline [35-39].

Hypertension has been associated with changes in brain structure and cognitive function [40-43]. It is known that hypertension accelerates the decline in cerebral perfusion $[44,45]$. Altered regional cerebral bloodflow in hypertensive subjects in the middle/posterior watershed areas has been found [45]. Moreover, memory performance in this group was related to this diminished response, so hypertension represents a risk for generating watershed cortical infarcts. Therefore, it is of importance to control for hypertension when studying brain structure, as we did.

In summary, our data show that patients with type 1 diabetes who had developed advanced retinopathy also had modest focal cortical atrophy on MRI of the brain. Prospective studies are warranted to establish whether reduced GMD in patients with diabetes is progressive, and how it affects various domains of cognitive functioning.

Acknowledgement This project was supported by a grant from the Dutch Diabetes Research Foundation (2001.11.012).

\section{References}

1. Reske-Nielsen E, Lundbaek K, Rafaelsen OJ (1965) Pathological changes in the central and peripheral nervous system of young longterm diabetics. Diabetologia 1:233-241

2. Brands AM, Biessels GJ, de Haan EH, Kappelle LJ, Kessels RP (2005) The effects of type 1 diabetes on cognitive performance: a meta-analysis. Diabetes Care 28:726-735

3. Ryan CM, Geckle MO, Orchard TJ (2003) Cognitive efficiency declines over time in adults with Type 1 diabetes: effects of micro- and macrovascular complications. Diabetologia 46:940-948

4. Ferguson SC, Blane A, Perros P et al (2003) Cognitive ability and brain structure in type 1 diabetes: relation to microangiopathy and preceding severe hypoglycemia. Diabetes 52:149-156

5. Dejgaard A, Gade A, Larsson H, Balle V, Parving A, Parving HH (1991) Evidence for diabetic encephalopathy. Diabet Med 8:162-167

6. Yousem DM, Tasman WS, Grossman RI (1991) Proliferative retinopathy: absence of white matter lesions at MR imaging. Radiology 179:229-230

7. Araki Y, Nomura M, Tanaka H et al (1994) MRI of the brain in diabetes mellitus. Neuroradiology 36:101-103
8. Lunetta M, Damanti AR, Fabbri G, Lombardo M, Di Mauro M, Mughini L (1994) Evidence by magnetic resonance imaging of cerebral alterations of atrophy type in young insulin-dependent diabetic patients. J Endocrinol Invest 17:241-245

9. Ashburner J, Friston KJ (2000) Voxel-based morphometry - the methods. Neuroimage 11:805-821

10. Wong TY, Klein R, Klein BE, Tielsch JM, Hubbard L, Nieto FJ (2001) Retinal microvascular abnormalities and their relationship with hypertension, cardiovascular disease, and mortality. Sury Ophthalmol 46:59-80

11. Patton N, Aslam T, Macgillivray T, Pattie A, Deary IJ, Dhillon B (2005) Retinal vascular image analysis as a potential screening tool for cerebrovascular disease: a rationale based on homology between cerebral and retinal microvasculatures. J Anat 206:319-348

12. Kwa VI, van der Sande JJ, Stam J, Tijmes N, Vrooland JL (2002) Retinal arterial changes correlate with cerebral smallvessel disease. Neurology 59:1536-1540

13. Aldington SJ, Kohner EM, Meuer S, Klein R, Sjolie AK (1995) Methodology for retinal photography and assessment of diabetic retinopathy: the EURODIAB IDDM complications study. Diabetologia 38:437-444

14. No authors listed (1997) Hypoglycemia in the Diabetes Control and Complications Trial. The Diabetes Control and Complications Trial Research Group. Diabetes 46:271-286

15. Good CD, Johnsrude IS, Ashburner J, Henson RN, Friston KJ, Frackowiak RS (2001) A voxel-based morphometric study of ageing in 465 normal adult human brains. Neuroimage $14: 21-36$

16. Senjem ML, Gunter JL, Shiung MM, Petersen RC, Jack CR Jr (2005) Comparison of different methodological implementations of voxel-based morphometry in neurodegenerative disease. Neuroimage 26:600-608

17. Friston K, Holmes AP, Worsley KJ, Poline JB (1995) Statistical parametric maps in functional imaging: a general linear approach. Hum Brain Mapp 2:189-210

18. Perros P, Deary IJ, Sellar RJ, Best JJ, Frier BM (1997) Brain abnormalities demonstrated by magnetic resonance imaging in adult IDDM patients with and without a history of recurrent severe hypoglycemia. Diabetes Care 20:1013-1018

19. Musen G, Lyoo IK, Sparks CR et al (2006) Effects of type 1 diabetes on gray matter density as measured by voxel-based morphometry. Diabetes 55:326-333

20. Brownlee M (2005) The pathobiology of diabetic complications: a unifying mechanism. Diabetes 54:1615-1625

21. Johnson PC, Brendel K, Meezan E (1982) Thickened cerebral cortical capillary basement membranes in diabetics. Arch Pathol Lab Med 106:214-217

22. de la Torre JC, Mussivand T (1993) Can disturbed brain microcirculation cause Alzheimer's disease? Neurol Res $15: 146-153$

23. Kalaria RN, Hedera P (1995) Differential degeneration of the cerebral microvasculature in Alzheimer's disease. Neuroreport $6: 477-480$

24. Pfefferbaum A, Mathalon DH, Sullivan EV, Rawles JM, Zipursky RB, Lim KO (1994) A quantitative magnetic resonance imaging study of changes in brain morphology from infancy to late adulthood. Arch Neurol 51:874-887

25. Tomlinson BE, Blessed G, Roth M (1970) Observations on the brains of demented old people. J Neurol Sci 11:205-242

26. Kaplan JG, Katzman R, Horoupian DS, Fuld PA, Mayeux R, Hays AP (1985) Progressive dementia, visual deficits, amyotrophy, and microinfarcts. Neurology 35:789-796

27. Munoz DG (1991) The pathological basis of multi-infarct dementia. Alzheimer Dis Assoc Disord 5:77-90

28. Kovari E, Gold G, Herrmann FR et al (2004) Cortical microinfarcts and demyelination significantly affect cognition in brain aging. Stroke 35:410-414

29. Snowdon DA, Greiner LH, Mortimer JA, Riley KP, Greiner PA, Markesbery WR (1997) Brain infarction and the clinical expression of Alzheimer disease. The Nun Study. JAMA 277:813-817 
30. Esiri MM, Wilcock GK, Morris JH (1997) Neuropathological assessment of the lesions of significance in vascular dementia. J Neurol Neurosurg Psychiatry 63:749-753

31. del Ser T, Bermejo F, Portera A, Arredondo JM, Bouras C, Constantinidis J (1990) Vascular dementia. A clinicopathological study. J Neurol Sci 96:1-17

32. Vinters HV, Ellis WG, Zarow C et al (2000) Neuropathologic substrates of ischemic vascular dementia. J Neuropathol Exp Neurol 59:931-945

33. Esiri MM (2000) Which vascular lesions are of importance in vascular dementia? Ann N Y Acad Sci 903:239-243

34. Lee JH, Olichney JM, Hansen LA, Hofstetter CR, Thal LJ (2000) Small concomitant vascular lesions do not influence rates of cognitive decline in patients with Alzheimer disease. Arch Neurol 57:1474-1479

35. Schofield PW, Logroscino G, Andrews HF, Albert S, Stern Y (1997) An association between head circumference and Alzheimer's disease in a population-based study of aging and dementia. Neurology 49:30-37

36. Obara K, Meyer JS, Mortel KF, Muramatsu K (1994) Cognitive declines correlate with decreased cortical volume and perfusion in dementia of Alzheimer type. J Neurol Sci 127:96-102

37. Meyer JS, Rauch GM, Crawford K et al (1999) Risk factors accelerating cerebral degenerative changes, cognitive decline and dementia. Int J Geriatr Psychiatry 14:1050-1061
38. de Leon MJ, George AE, Reisberg B et al (1989) Alzheimer's disease: longitudinal CT studies of ventricular change. AJR Am J Roentgenol 152:1257-1262

39. DeCarli C, Haxby JV, Gillette JA, Teichberg D, Rapoport SI, Schapiro MB (1992) Longitudinal changes in lateral ventricular volume in patients with dementia of the Alzheimer type. Neurology 42:2029-2036

40. Salerno JA, Murphy DG, Horwitz B et al (1992) Brain atrophy in hypertension. A volumetric magnetic resonance imaging study. Hypertension 20:340-348

41. Strassburger TL, Lee HC, Daly EM et al (1997) Interactive effects of age and hypertension on volumes of brain structures. Stroke 28:1410-1417

42. Yamano S, Sawai F, Yamamoto Y et al (1999) Relationship between brain atrophy estimated by a longitudinal computed tomography study and blood pressure control in patients with essential hypertension. Jpn Circ J 63:79-84

43. Taki Y, Goto R, Evans A et al (2004) Voxel-based morphometry of human brain with age and cerebrovascular risk factors. Neurobiol Aging 25:455-463

44. Meyer JS, Rauch G, Rauch RA, Haque A (2000) Risk factors for cerebral hypoperfusion, mild cognitive impairment, and dementia. Neurobiol Aging 21:161-169

45. Jennings JR, Muldoon MF, Ryan C et al (2005) Reduced cerebral blood flow response and compensation among patients with untreated hypertension. Neurology 64:1358-1365 